Pacific Journal of Mathematics

FINITELY GENERATED PROJECTIVE EXTENSIONS OF 


\title{
FINITELY GENERATED PROJECTIVE EXTENSIONS OF UNIFORM ALGEBRAS
}

\author{
JOAN VERDERA
}

\begin{abstract}
Let $A$ and $B$ be uniform algebras and suppose that $B$ is an extension of $A$, finitely generated and projective as an $A$-module. Let $\pi$ denote the natural projection from the maximal ideal space of $B$ onto the maximal ideal space of $A$. We show that $K$ is a generalized peak interpolation set for $B$ if and only if $\pi(K)$ is a generalized peak interpolation set for $A$. Then we give a topological description of the maximals sets of antisymmetry of $B$ in terms of those of $A$. Finally, we prove that if $B$ is strongly separable over $A$, then the algebra of $B$-holomorphic functions is strongly separable over the algebra of $A$-holomorphic functions.
\end{abstract}

1. Introduction. The main motivation for this work comes from a series of results discovered over the last twenty years concerning the structure of certain types of integral extensions of (commutative complex unital) Banach algebras. More precisely, the results which we are refering to group roughly in two classes. On one hand, we have the theory of the so-called algebraic or ArensHoffman extensions [1, 2, 7, 8]. These are extensions of the form $A[x] /(\alpha(x))$, where $\alpha(x)$ is a monic polynomial with coefficients in the base algebra $A$. Moreover, part of this theory was recently extended [12] to the case where the extension is finitely generated and projective as an $A$-module. On the other hand, we have results coming from the study of strongly separable extensions [4, 9], i.e., extensions which are finitely generated and projective as $A$-modules and separable as $A$-algebras.

Still a word on method. As we showed in [12], given a finitely generated projective extension $B$ of a Banach algebra $A$ and an element $b \in B$, one can pick out, among all monic polynomials $\alpha(x) \in$ $A[x]$ such that $\alpha(b)=0$, a canonical one, which enjoys some useful properties (see Lemma 1 below for a precise statement). Then, our method consists in obtaining information about $B$ from information about $A$ (and conversely) by means of these canonical integrity equations.

We fix now some notation. $M_{1}$, and $\partial_{1}$, denote, respectively, the character spectrum and the Shilov boundary operators on Banach algebras; $\hat{f}$ is the Gelfand transform of $f$. $A$ will denote a fixed uniform algebra on a compact space $X$, and $B$ a finitely generated projective extension of $A$. It is known that $B$ can be endowed with a canonical Banach algebra structure [9, Th. 4, p. 138 and 12, §3]. 
However, for our purposes, it will not be necessary to handle any specific Banach algebra norm on $B$ and only the existence of such a norm will be required. We will also assume, except in Theorem 1 , that $B$ is a uniform algebra.

We write $\pi$ for the projection from $M_{B}$ onto $M_{A}$ defined $\pi(\psi)=$ $\left.\dot{\psi}\right|_{A}, \dot{\psi} \in M_{B}$.

If $\alpha(x)=\sum_{i=0}^{n} \alpha_{i} x^{i}$ is a polynomial with coefficients in $A$ and $\dot{\phi} \in M_{\cdot A}$, then we write

and

$$
\alpha_{\phi}(x)=\sum_{i=0}^{n} \phi\left(\alpha_{i}\right) x^{i} \in C[x]
$$

$$
Z\left(\alpha_{\dot{\phi}}\right)=\left\{\lambda \in \boldsymbol{C}: \alpha_{\dot{\phi}}(\lambda)=0\right\} .
$$

Our arguments are based on the following result from [12]:

Lemma 1. Assume that $B$ has a well defined rank over $A$, say n. Then for each $b \in B$ there exists a monic polynomial $\alpha(x) \in A[x]$ of degree $n$ such that $\alpha(b)=0$ and

$$
Z\left(\alpha_{\phi}\right)=\hat{b}\left(\pi^{-1}(\phi)\right), \quad \phi \in M_{i d} .
$$

The notation and terminology we use are standard (see [6] and [11]). For basic facts about projective modules and (algebraically) separable algebras the reader is referred to [5].

2. Peak interpolation sets. Before stating our first result we recall some well known definitions.

Let $\mathfrak{B}$ be a Banach algebra, $Z$ a closed boundary for $\mathfrak{B}$ and $K$ a closed subset of $Z$. Then $K$ is said to be a peak set for $\mathfrak{B}$ on $Z$ if there exists $f \in \mathfrak{B}$ such that $\hat{f}=1$ on $K$ and $|\hat{f}|<1$ on $Z \backslash K$. If $K$ is an intersection of peak sets for $\mathfrak{B}$ on $Z$, we say that $K$ is a generalized peak set for $\mathfrak{B}$ on $Z$. We call $K$ an interpolation set for $\mathfrak{B}$ if, given any $h \in C(K)$, there exists $f \in \mathfrak{B}$ such that $\hat{f}=h$ on $K$. If $K$ is both a (generalized) peak set for $\mathfrak{B}$ on $Z$ and an interpolation set for $\mathfrak{B}$, then it is called a (generalized) peak interpolation set for $\mathfrak{B}$ on $Z$. When $\mathfrak{B}$ is a uniform algebra on $Z$, the explicit reference to $Z$ is usually dropped, so that one simply speaks of (generalized) peak (resp. peak interpolation) sets for $\mathfrak{B}$.

As we said before, in the following theorem we just assume that $B$ is a finitely generated projective extension of $A$, endowed with some Banach algebra structure. The corollary in $[12, \S 2]$ implies that $Y=\pi^{-1}(X)$ is a closed boundary for $B$.

THEOREM 1. If $K \subset Y$ is a generalized interpolation set for $B$ on $Y$, then $\pi(K)$ is a generalized peak interpolation set for $A$. 
CoRollaRy. If $K \subset Y$ is a peak interpolation set for $B$ on $Y$, then $\pi(K)$ is a peak interpolation set for $A$.

This corollary follows immediately from the theorem and from the fact that $\pi$ preserves $G_{i}$-sets.

Proof of Theorem 1. Without loss of generality, we may assume $B$ to have a well defined rank over $A$. This is so because there are mutually orthogonal idempotents $e_{1}, \cdots, e_{p}$ in $A$ such that $e_{1}+\cdots+$ $e_{p}=1$ and, for each $i, e_{i} B$ is a finitely generated projective extension of $e_{i} A$, with a well defined rank over $e_{i} A$ [5, 4.11, p. 31].

Let $B_{0}$ be the uniform closure in $C\left(M_{B}\right)$ of $\hat{B}$. Then $M_{B_{0}}=M_{B}$ and $\partial_{B_{0}}=\partial_{B}$. Moreover, as it is easily seen, $B_{0}$ satisfies the conclusion of Lemma 1 . We shall regard $B_{0}$ as a uniform algebra on $Y$ and we shall prove the theorem under the weaker hypothesis that $K$ is a generalized peak interpolation set for $B_{0}$.

If $H=\pi(K)$, then, by the Bishop-Glicksberg characterization of generalized peak interpolation sets $[11,20.10$, p. 210], we have to show that $|\mu|(H)=0$ for any complex regular Borel measure $\mu$ on $X$ orthogonal to $A$. In order to see this, it is clearly sufficient to prove the following:

$$
\text { for each } \phi \in H \text { there exists a closed neighborhood (in }
$$

$X) U_{0}$ of $\phi$ such that $|\mu|\left(U_{0} \cap H\right)=0$.

Fix $\phi \in H$ and let $\psi_{1}, \cdots, \psi_{m}$ be the different points in $\pi^{-1}(\phi)$. Apply the structure theorem for $\pi$ [12, Th. 1] to find mutually disjoint open neighborhoods (in $Y$ ) $V_{1}, \cdots, V_{m}$ of $\psi_{1}, \cdots, \psi_{m}$ and an open neighborhood (in $X$ ) $U$ of $\phi$ such that

$$
\pi\left(V_{i}\right)=U \text { for each } i, \pi^{-1}(U)=\bigcup_{i} V_{i}
$$

and

$$
m\left(\psi_{i}\right)=\sum_{\psi \in \pi^{-1}(\theta) \cap v_{i}} m(\psi), \quad \theta \in U, \quad 1 \leqq i \leqq m
$$

where $m(\cdot)$ is the multiplicity function defined in $\S 1$ of [12].

Let $U_{0}$ be a compact neighborhood of $\phi$, contained in $U$. We have $U_{0} \cap H=\bigcup_{i} \pi\left(K \cap \pi^{-1}\left(U_{0}\right) \cap V_{i}\right)$, and so, it order to prove (2), we can assume that $K$ is included in a $V_{i}$, say $V_{1}$.

We claim now that there exists $g \in B_{0}$ satisfying

$$
g\left(\pi^{-1}(H) \cap V_{1}\right)=\{1\} \text { and } g\left(\pi^{-1}(H) \cap V_{i}\right)=\{0\}, \quad i>1 .
$$

To prove the claim, write $I=\{f \in A: f(H)=\{0\}\}, J=\left\{f \in B_{0}\right.$ : $f(K)=\{0\}\}$, and observe that $B_{0} / J$ is an integral extension of $A / I$. 
Hence, each character in $M_{A / I}$ is the restriction to $A / I$ of a character of $B_{0} / J[14$, p. 259]. On the other hand, our hypothesis on $K$ implies that $B_{0} / J \cong C(K)$, and thus

$$
H=\pi(K)=\pi\left(M_{B_{0} / J}\right)=M_{A / I} .
$$

Now, $B / I B$ is a finitely generated projective extension of $A / I$ and so it is a Banach algebra under some norm. Since $M_{B / I B}=\pi^{-1}(H)$ (because $H=M_{A / I}$ ) and $\pi^{-1}(H)$ is the disjoint union of the closed subsets $\pi^{-1}(H) \cap V_{i}$, the Shilov idempotent theorem [10, 8.9, p. 73] can be applied to get $g \in \hat{B}$ satisfying (4).

To end the proof we still need some auxiliary tools. For $\psi \in M_{B}$ and for each neighborhood $V$ (in $M_{B}$ ) of $\psi$, we define

$$
c_{\psi}(V)=\max _{\theta \in \pi(V)} \operatorname{card}\left(\pi^{-1}(\theta) \cap V\right)
$$

and

$$
r(\psi)=\min _{V} c_{\psi}(V)
$$

where $V$ ranges over all neighborhoods of $\psi$. The number $r(\psi)$ may be interpreted as a ramification index for $\pi$ at $\psi$.

For $\theta \in H$ we define

$$
N(\theta)=\max _{\psi \in \pi^{-1}(\theta) \cap K} r(\psi) .
$$

Now we can complete the proof of (2). We will proceed by induction on $N(\phi)$.

Suppose $N(\phi)=1$. In this case, we will prove directly that $H$ is a generalized peak interpolation set for $A$. As $r\left(\psi_{1}\right)=1$, shrinking $V_{1}$ and $U$ if necessary, we can also assume that $\pi \mid V_{1}$ is an homeomorphism onto $U$. Given an open neighborhood $W$ of $H, W \subset U$, and given $\varepsilon>0$, then, by hypothesis, there is a peak set $K^{\prime}$ for $B_{0}$ such that $K \subset K^{\prime} \subset \pi^{-1}(W)$. Choose $f \in B_{0}$ which peaks on $K^{\prime}$ and satisfies

$$
|f(\psi)|<\varepsilon / n\|g\|_{Y}, \quad \psi \in Y \backslash \pi^{-1}(W)
$$

and then consider the function $h$ defined by

$$
h(\theta)=\sum_{\psi \in \pi^{-1}(\theta)} m(\psi)(f g)(\psi), \quad \theta \in X
$$

If $\alpha(x)=\sum_{i=0}^{n} \alpha_{i} x^{i}$ is a polynomial related to $b=f g$ as in Lemma 1 , then $h=-\alpha_{n-1} \in A$ by (1). Writing $k=h / m\left(\psi_{1}\right)$, we obtain

$$
\begin{aligned}
k(\theta) & =1, \quad \theta \in H \\
|k(\theta)| & \leqq \varepsilon, \quad \theta \in X \backslash W \\
\|k\|_{X} & \leqq n\|g\|_{Y} .
\end{aligned}
$$


Thus $H$ is a generalized peak set for $A$.

Let $h_{0} \in C(H)$. If $f \in B_{0}$ is such that $f(\psi)=h_{0}(\pi(\psi)), \psi \in K$, and $k$ is constructed from $f$ and $g$ as above, then $\left.k\right|_{H}=h_{0}$ and $k \in A$.

Now, assume that $N(\phi)>1$ and that (2) holds for those $\theta \in H$ with $N(\theta)<N(\phi)$. Since $N(\phi)=r\left(\psi_{1}\right)$, we may suppose that $N(\phi)=$ $c_{\psi_{1}}\left(V_{1}\right)$. If we put

$$
H_{1}=\left\{\theta \in H: r(\psi)<r\left(\psi_{1}\right) \text { for each } \psi \in \pi^{-1}(\theta) \cap K\right\}
$$

and

$$
H_{0}=\left\{\theta \in H: \pi^{-1}(\theta) \cap V_{1} \text { is a singleton }\right\},
$$

then $|\mu|\left(H_{1}\right)=0$ by the inductive hypothesis. Moreover, $H_{0}$ is closed and $H=H_{0} \cup H_{1}$. We claim now that $H_{0}$ is a generalized peak interpolation set for $A$. To see this, observe that $K_{0}=\pi^{-1}\left(H_{0}\right) \cap K$ is a generalized peak interpolation set for $B_{0}$ and that $\pi$ is an homeomorphism from $K_{0}$ onto $H_{0}$. Then, the same argument as above can be used to deduce the desired conclusion. Thus, $|\mu|\left(H_{0}\right)=0$ and the proof is complete.

REMARKs. (a) The projectivity hypothesis on $B$ cannot be relaxed, as shown by the following example.

ExAmple 1. Let $B$ the disk algebra, and put $A=\{f \in B: f(0)=$ $f(1)$. Then $B$ is an extension of $A$, finitely generated as an $A$ module, but 1 is not a peak point for $A$, although it is a peak point for $B$.

(b) It would be interesting to find out whether Theorem 1 is true when $A$ is a semisimple Banach algebra, and also whether an analogous statement for peak sets holds. In both cases, the main difficulty seems to be the reduction to a local statement.

From now on, $B$ will be assumed to be a uniform algebra on $Y$. Recall [12, Th. 3] that this is the case if and only if $m(\psi)=1$, $\psi \in \partial_{B}$, and then, in particular, $\partial_{B}$ is a covering space of $\partial_{A}$ with projection $\pi$.

THEOREM 2. Let $K$ be a subset (resp. $a G_{i}$ subset) of $Y$. Then, $K$ is a generalized peak interpolation (resp. a peak interpolation) set for $B$ if and only if $\pi(K)$ is a generalized peak interpolation. (resp. a peak interpolation) set for $A$.

Proof. The "only if part" is contained in Theorem 1.

Assume $H=\pi(K)$ to be a generalized peak interpolation set for $A$. If $I=\{f \in A: f(H)=\{0\}\}$, then $B / I B$ is a finitely generated pro- 
jective extension of $A / I=C(H)$. Since $m(\psi)=1, \psi \in \partial_{B}$ and $H \subset \partial_{A}$, we can write

$$
m(\psi)=1, \quad \psi \in \pi^{-1}(H)=M_{B / I B} .
$$

But this means that $B / I B$ is separable as $C(H)$-algebra (use [5, Th. 7.1, (c) $\rightarrow$ (a), p. 72]), and hence, applying [3, Th. 2, p. 30], we conclude that $B / I B \cong C\left(\pi^{-1}(H)\right)$. As $\pi^{-1}(H)$ is obviously a generalized peak set for $B$, the proof is complete.

CoRollary. If $\gamma_{A}$ and $\gamma_{B}$ denote the Choquet boundaries of $A$ and $B$ respectively, then $\gamma_{B}=\pi^{-1}\left(\gamma_{A}\right)$.

REMARKS. (a) The "if part" of Theorem 2 is not true without uniformity assumptions on $B$. For example, if $A=C[0,1]$ and $B=$ $A[x] /\left(x^{2}-f\right)$, where $f(t)=t$ for each $t \in[0,1]$, then $B$ is not a uniform algebra according to [12, Th. 3]. Therefore, $Y$ itself is not an interpolation set for $B$.

(b) The statement in the "only if part" of Theorem 2 is not true for interpolation sets as shown by the following example.

EXAMPLE 2. Consider a uniform algebra $A$ with the following property (for example the disc algebra): there exist interpolation sets $K_{0}, K_{1}$ for $A$ such that $K_{0} \cup K_{1}$ is not an interpolation set for $A$. Put $B=A[x] /\left(x^{2}-1\right)$, so that we may identify $M_{B}$ with $M_{A} \times\{0,1\}$. Now, $K=\mathrm{U}_{j=0}^{1} K_{j} \times\{j\}$ is an interpolation set for $B$, but $\pi(K)=$ $K_{0} \cup K_{1}$ is not an interpolation set for $A$.

(c) The arguments used in the proof of Theorem 2 can be applied to deal with some examples arising from the theory of several complex variables.

EXAMPle 3. Write

$$
D_{n}=\left\{z \in C^{p}:\left|z_{1}\right|^{2}+\cdots+\left|z_{p-1}\right|^{2}+\left|z_{p}\right|^{2 n} \leqq 1\right\},
$$

$n$ a positive integer. Then

$$
\begin{aligned}
& D_{n} \stackrel{\pi}{\longrightarrow} D_{1} \\
& z \longrightarrow\left(z_{1}, \cdots, z_{p-1}, z_{p}^{n}\right)
\end{aligned}
$$

is an $n$-sheeted covering map, ramified along $\left\{z: z_{p}=0\right\}$. Let $A$ (resp. $B$ ) be the algebra of continuous functions on $D_{1}$ (resp. $D_{n}$ ) which are holomorphic in the interior of $D_{1}$ (resp. $D_{n}$ ). We claim that Theorem 2 is true in this case. To prove the claim, take first a peak interpolation set $K$ for $B$. Then $K$ is a zero set for $B$, that is, there is $f \in B$ with $K=f^{-1}(0)$. Since Lemma 1 also works in this case, $\pi(K)$ 
is a zero set for $A$, and hence a peak interpolation set for $A$ [13, Th. 1.1, p. 484]. Assume now that $K$ is a peak interpolation set for $A$. Since $\pi$ is a local homeomorphism on $D_{n} \mid\left\{z: z_{p}=0\right\}$, one can prove that $\pi^{-1}(H)$ is a peak interpolation set for $B$ for each compact subset $H$ of $K \backslash\left\{z: z_{p}=0\right\}$. But on $K \cap\left\{z: z_{p}=0\right\} B$ and $A$ are the same algebra, thus $\pi^{-1}(K)$ is a peak interpolation set for $B$.

In particular, we have shown that on $\partial B$ zero sets, peak sets and peak interpolation set are the same, although, for $n>1$ and $p>1$, the interior of $D_{n}$ is not strictly pseudoconvex.

\section{Antisymmetric decompositions.}

LeMma 2. If $A$ is an antisymmetric algebra, then the maximal sets of antisymmetry for $B$ on $M_{B}$ are the connected components of $M_{B}$.

Proof. We show first that if $M_{B}$ is connected, then $B$ is antisymmetric. To see this, let $f \in B$ and assume $\hat{f}$ to be a real function. Since $A$ is antisymmetric, there are not nontrivial idempotents in $A$, and thus $B$ has a well defined rank over $A[5,4.12, \mathrm{p} .32]$, say $n$. If $\alpha(x)=\sum_{i=0}^{n} \alpha_{i} x^{i}$ is a polynomial obtained from $f$ by applying Lemma 1 , then each $\hat{\alpha}_{i}$ is a real function (by (1)), so constant. Therefore, $\hat{f}\left(M_{B}\right)$ is finite, but since $M_{B}$ is connected, $\hat{f}\left(M_{B}\right)$ is in fact a point.

Consider now $m$ different connected components of $M_{B}$, say $C_{1}, \cdots, C_{m}$, and separate them by means of mutually disjoint open and closed sets $U_{1}, \cdots, U_{m}$. As $M_{A}$ is connected, one has $\pi\left(U_{i}\right)=M_{A}$ for each $i$, and this implies that $m \leqq n$. Thus, $M_{B}$ has only a finite number of connected components $C_{1}, \cdots, C_{m}$, which are open and closed sets. Let $e_{i}$ be the idempotent in $B$ whose Gelfand transform is the characteristic function of $C_{i}$. The first part of the proof, applied to $e_{i} B$, tells us that $C_{i}$ is a set of antisymmetry for $B$. If $C_{i} \varsubsetneqq S$, then $\hat{e}_{i}$ is real and nonconstant on $S$, so $C_{i}$ is, in fact, a maximal set of antisymmetry for $B$ on $M_{B}$.

THEOREM 3. Let $\left(K_{i}\right)_{i \in I}$ be the family of maximal sets of antisymmetry for $A$ on $X$. Then the family of maximal sets of antisymmetry for $B$ on $Y$ is $\left(C_{i j} \cap Y\right)_{i, j}$, where, for each $i \in I,\left(C_{i j}\right)_{1 \leq j \leq p_{i}}$ is the collection of connected components of $\pi^{-1}\left(\hat{K}_{i}\right), \hat{K}_{i}$ being the $A$ convex hull of $K_{i}$. Moreover, $\sup _{i \in I} p_{i}<\infty$.

Proof. Assume first that $X=M_{A}$, so that $K_{i}=\hat{K}_{i}, i \in I$. For each $i \in I, A_{i}=\left\{\left.f\right|_{K_{i}}: f \in A\right\}$ is an antisymmetric uniform algebra, $M_{A i}=K_{i}$ and $\partial_{A i} \subset K_{i} \cap \partial_{A}\left[6\right.$, Th. 11 (c), p. 167]. If we set $I_{i}=$ 
$\left\{f \in A: f\left(K_{i}\right)=\{0\}\right\}$, then $B_{i}=B / I_{i} B$ is a finitely generated projective extension of $A / I_{i} \cong A_{i}$ and $M_{B_{i}}=\pi^{-1}\left(K_{i}\right)$. By the corollary in $\S 2$ of [12], we have

$$
\partial_{B_{i}}=\pi^{-1}\left(\partial_{A i}\right) \subset \pi^{-1}\left(\partial_{A}\right)=\partial_{B} .
$$

Now, since $B$ is a uniform algebra, we get [12, Th. 3]

$$
m(\psi)=1, \quad \psi \in \partial_{B}
$$

and thus, in particular, $m(\psi)=1, \psi \in \partial_{B_{i}}$. Again appealing to [12, Th. 3], we conclude that $B_{i}$ is a uniform algebra, hence that $B_{i}=$ $\left\{f \mid \pi-1_{\left(K_{i}\right)}: f \in B\right\}$.

By Lemma 2, for each $j \in\left\{1,2, \cdots, p_{i}\right\}, C_{i j}$ is a set of antisymmetry for $B$. Let $S$ be the maximal set of antisymmetry for $B$ on $M_{B}$ containing $C_{i j}$. If $\pi(S) \neq K_{i}$, then there would exist an $a \in A$ which would be real and nonconstant on $\pi(S)$. But then $a \in B$ would be real and nonconstant on $S$, which is impossible. So $\pi(S)=K_{i}$. From this we obtain $C_{i j} \subset S \subset \pi^{-1}\left(K_{i}\right)$, and, since $S$ is connected, we get $S=C_{i j}$.

In order to prove the general case, notice that $\left(\hat{K}_{i}\right)_{i \in I}$ is the family of maximal sets of antisymmetry for $A$ on $M_{A}[6, \mathrm{Th} .15$, p. 171]. But the maximal sets of antisymmetry for $B$ on $Y$ are the intersection with $Y$ of these for $B$ on $M_{B}$ [6, Th. 14, p. 171], and thus from the first part of the proof we can draw out the desired conclusion.

In the following theorem we write $E_{A}$ (resp. $E_{B}$ ) for the essential set of $A$ (resp. $B$ ).

THEOREM 4. We have $E_{B}=\pi^{-1}\left(E_{A}\right)$. In particular, $B$ is essential if and only if $A$ is essential.

Proof. One can prove, using an elementary argument based on the structure theorem for $\pi$, that

$$
\pi^{-1}(\mathrm{Cl} F)=\mathrm{Cl} \pi^{-1}(F), \quad \text { for each } F \subset X,
$$

where $\mathrm{Cl}$ denotes topological closure.

Let $P_{A}$ (resp. $P_{B}$ ) be the union of all one point maximal sets of antisymmetry for $A$ (resp. $B$ ), so that, by Theorem $3, P_{B}=\pi^{-1}\left(P_{A}\right)$. Using (5) and [6, Corollary 2, p. 65] we obtain

$$
E_{B}=\mathrm{Cl}\left(\pi^{-1}\left(X \backslash P_{A}\right)\right)=\pi^{-1}\left(\mathrm{Cl}\left(X \backslash P_{A}\right)\right)=\pi^{-1}\left(E_{A}\right) .
$$

4. B-holomorphic functions. If $\mathfrak{B}$ is a uniform algebra and $U$ an open subset of $M_{\mathfrak{x}}$, then a complex function on $U$ is called a 
locally $\mathfrak{B}$-approximable function if each point in $U$ has a neighborhood on which $f$ is uniformly approximable by functions in $\mathfrak{B}$. We write $L_{\mathfrak{g}}$ for the algebra of the locally $\mathfrak{B}$-approximable functions defined on all $M_{\mathfrak{B}}$, and we denote by $H_{\mathfrak{B}}$ the smallest subalgebra of $C\left(M_{\mathfrak{3}}\right)$ which contains $\mathfrak{B}$ and is closed under local uniform approximation.

At this point, some remarks on the relation between $L_{\mathfrak{B}}$ and $H_{\mathfrak{B}}$ are in order. A simple inductive argument [10, 8.1, p. 19] shows the following:

There exists an ordinal $\mu$ such that to each ordinal $\nu \leqq \mu$ there corresponds a subalgebra $H_{\mathfrak{3}}^{\nu}$ of $C\left(M_{\mathfrak{B}}\right)$ with the following properties:

(i) $H_{\mathfrak{9}}^{0}=\mathfrak{B}, H_{\mathfrak{B}}^{\mu}=H_{\mathfrak{B}}$, and $H_{\mathfrak{B}}^{\alpha} \varsubsetneqq H_{\mathfrak{B}}^{\beta}$ for $0 \leqq \alpha<\beta \leqq \mu$.

(ii) If $0<\nu \leqq \mu$ then $H_{\Re}^{\nu}=L_{\mathfrak{B}_{\nu}}$, where $\mathfrak{B}_{\nu}$ is the uniform closure in $C\left(M_{\mathfrak{B}}\right)$ of the algebra $\mathrm{U}_{\alpha<\nu} H_{\mathfrak{3}}^{\alpha}$.

Let us observe that, by a theorem of Rickart [10, 40.3, p. 116], we have $M_{\mathfrak{B}_{\nu}}=M_{\mathfrak{B}}$ for each $\nu$, so that, according to (ii) and to our notational conventions, $H_{\mathfrak{B}}^{\nu}$ is a subalgebra of $C\left(M_{\mathfrak{B}}\right)$.

Functions in $H_{\mathfrak{F}}$ (resp. $H_{\mathfrak{3}}$ ) are called $\mathfrak{B}$-holomorphic functions (resp. $\mathfrak{B}$-holomorphic functions of class $\nu$ ) by Rickart [10, §17].

THEOREM 5. If $B$ is a strongly separable A-algebra, then

(a) $H_{B}$ (resp. $L_{B}$ ) is a strongly separable $H_{A}$ (resp. $L_{A}$ )-algebra.

(b) If $A=H_{A}$ (resp. $A=L_{A}$ ), then $B=H_{B}$ (resp. $B=L_{B}$ ).

We divide the proof of Theorem 5 into three lemmas.

Let $S$ be an extension of a commutative unital ring $R$. If $S$ is finitely generated and projective as an $R$-module, then one can define a distinguished $R$-module homomorphism from $S$ into $R$, called the trace map. Then one proves $[5,2.1$, p. 92]:

LEMMA 3. The extension $S$ of the commutative unial ring $R$ is strongly separable over $R$ if and only if there is an $R$-module homomorphism $t$ from $S$ into $R$ and elements of $S x_{1}, \cdots, x_{m} ; y_{1}, \cdots, y_{m}$ with

(i) $\sum x_{j} y_{j}=1$

(ii) $x=\sum_{j} t\left(x y_{j}\right) x_{j}, x \in S$.

Moreover the map $t$ is always the trace map from $S$ to $R$.

LeMma 4. If $B$ is a strongly separable A-algebra and has a well defined rank over $A$, then for each $\phi \in M_{A}$ there exists a monic polinomial $\alpha(x) \in A[x]$ and $a d \in A$ with $d(\phi) \neq 0$, such that $B_{d}$ is isomorphic, as an $A_{d}$-algebra, to $A_{d}[x] /\left(\alpha(x)\right.$ ) (here $A_{d}$ and $B_{d}$ stand for the 
quotient rings of the multiplicative system of the powers of $d)$.

Proof. Given $\phi \in M_{A}$, consider an element $b_{0}$ of $B$ which separates the points of $\pi^{-1}(\phi)$, and a polynomial $\alpha(x) \in A[x]$ given by Lemma 1 applied to $b_{0}$. Define $d$ as the discriminant of $\alpha(x)$, so that $d(\phi) \neq 0$. Let $\varphi$ be the unique $A_{d}$-algebra homomorphism from $A_{d}[x] /(\alpha(x))$ into $B_{d}$ satisfying $\varphi([x])=b_{0} / 1$, where $[x]$ is the class of the polynomial $x$.

Suppose that, for certain nonnegative integers $m_{i}$,

$$
\sum_{i=0}^{n-1}\left(a_{i} / d^{m_{i}}\right)\left(b_{0}^{i} / 1\right)=0, \quad a_{i} \in A, \quad n=\operatorname{rank}_{A} B .
$$

Then, for a large nonnegative integer $m$,

$$
\sum_{i=0}^{n-1} d^{m} a_{i} b_{0}^{i}=0
$$

If $d(\omega) \neq 0, \omega \in M_{A}$, then the complex polynomial

$$
\sum_{i=0}^{n-1} d^{m}(\omega) \alpha_{i}(\omega) x^{i}
$$

is annihilated by the $n$ elements of $b_{0}\left(\pi^{-1}(\omega)\right)$. From this we obtain $d a_{i}=0,0 \leqq i \leqq n-1$. Thus

$$
a_{i} / d^{m_{i}}=0 \text { in } A_{d}, \quad 0 \leqq i \leqq n-1,
$$

that is, $\varphi$ is injective.

Write $B_{0}=\operatorname{Im} \varphi$. Then $B_{0}$ is a strongly separable $A_{d}$-algebra [5, Problem 8, p. 85] with a well defined rank $n$ over $A_{d}$. Since $B_{d}$ is a finitely generated projective $A_{d}$-module, we conclude, owing to the lifting property of projective modules over separable algebras [5, 2.3, p. 48], that $B_{d}$ is a finitely generated projective $B_{0}$-module. But the rank of $B_{d}$ over $A_{d}$ is $n$, so the rank of $B_{d}$ over $B_{0}$ is 1 , and thus $B_{d}=B_{0}$. Therefore $\varphi$ is an isomorphism.

Lemma 5. With the same hypothesis on $B$ as in Lemma 4, the following holds:

If $V, U$ are open set in $M_{B}$ and $M_{A}$ respectively, and if $\pi \mid V$ is an homeomorphism onto $U$, then, for each $b \in B, \beta=b \circ(\pi \mid V)^{-1}$ is locally A-approximable on $U$.

Proof. Consider $\phi \in U$ and let $b_{0}, \alpha(x)$ and $d$ be as in the proof of Lemma 4. Shrinking $U$ and $V$ we may assume that $d(\omega) \neq 0$ for each $\omega \in U$.

By Lemma 4, we have 


$$
\beta=\sum_{i=0}^{n-1}\left(a_{i} / d^{m_{i}}\right) \beta_{0}^{i}
$$

where $\beta_{0}=b_{0} \circ(\pi \mid V)^{-1}, a_{i} \in A$ and $m_{i}$ is a nonnegative integer for each $i$.

A standard argument shows that $d^{-1}$ is a locally $A$-approximable function on $U$. That $\beta_{0}$ is also locally $A$-approximable on $U$ follows from the fact that, $d$ never being zero on $U, \beta_{0}$ may be locally expressed as a uniformly convergent power series in the coefficients of $\alpha(x)$.

Proof of Theorem 5. According to Lemma 3 there exist elements of $B x_{1}, \cdots, x_{m} ; y_{1}, \cdots, y_{m}$ such that $\sum x_{i} y_{i}=1$ and

$$
t(f)=\sum_{i} t\left(f y_{i}\right) x_{i}, \quad f \in B
$$

We may assume $B$ to have a well defined rank $n$ over $A$ (use the argument in the first paragraph of the proof of Theorem 1). This assumption and the strong separability of $B$ imply that $M_{B}$ is an $n$-sheeted covering space of $M_{A}$ with projection $\pi$ [9, Th. 5, p. 138]. In this context, the trace map is given by

$$
t(f)(\phi)=\sum_{\psi \in \pi^{-1}(\phi)} f(\psi), \quad f \in B, \quad \phi \in M_{A} .
$$

But $C\left(M_{B}\right)$ is a strongly separable extension of $C\left(M_{A}\right)$ [3, Th. 2, p. 30] and, consequently, its trace map is given, for $f \in C\left(M_{B}\right)$, by (7). Moreover, relation (6) is true for $f \in C\left(M_{B}\right)$. Therefore, by Lemma 3 , we only have to show that

$$
t(f) \in H_{A} \quad \text { if } f \in H_{B} .
$$

We will prove, by transfinite induction, that

$$
t(f) \in H_{A}^{\nu} \text { if } f \in H_{B}^{\nu}, \quad \text { for each } \nu .
$$

If $\nu>0$ is an ordinal such that (9) is true for all $\alpha<\nu$, then clearly we have

$$
t(f) \in A_{\nu} \quad \text { if } \quad f \in B_{\nu} .
$$

Therefore, replacing $A_{\nu}$ and $B_{\nu}$ by $A$ and $B$, we are led to prove that

$$
t(f) \in L_{A} \quad \text { if } \quad f \in L_{B} .
$$

Given $f \in L_{B}$, fix $\phi \in M_{A}$ and consider mutually disjoint open neighborhoods $V_{i}$ of the points in $\pi^{-1}(\phi)$, and an open neighborhood $U$ of $\phi$ such that 
(i) $\pi \mid V_{i}$ is an homeomorphism onto $U$ for each $i$.

(ii) $f$ is uniformly approximable on each $V_{i}$ by functions in $B$. Given $\varepsilon>0$, consider $b_{i} \in B$ with $\left\|f-b_{i}\right\|_{v_{i}}<\varepsilon, 1 \leqq i \leqq n$. Thus

$$
\left\|t(f)-\sum_{i=1}^{n} \beta_{i}\right\|_{U}<n \varepsilon,
$$

where $\beta_{i}=b_{i} \circ\left(\pi \mid V_{i}\right)^{-1}, 1 \leqq i \leqq n$. Now Lemma 5 says that $t(f) \in L_{A}$. This completes the induction and so the proof of (8).

REMARK. The above proof shows that a slightly more general statement is true. In fact, we have proved that $H_{B}^{\nu}$ is a strongly separable $H_{A}^{\nu}$-algebra and that $A=H_{A}^{\nu}$ implies $B=H_{B}^{\nu}$ for each ordinal $\nu$.

Finally, we point out two problems whose solution we do not know.

PRoblem 1. In the standard hypothesis of this paper, that is, $A \subset B, A$ and $B$ uniform algebras, $B$ finitely generated and projective as an $A$-module, is $H_{B}$ (resp. $L_{B}$ ) finitely generated and projective as an $H_{A}$ (resp. $L_{A}$ )-module?

Problem 2. With the same hypothesis on $A$ and $B$, does $A=$ $H_{A}$ (resp. $A=L_{A}$ ) imply $B=H_{B}$ (resp. $\left.B=L_{B}\right)$ ?.

Acknowledgment. The author is grateful to the referee for some useful remarks and suggestions.

\section{REFERENCES}

1. R. Arens and K. Hoffman, Algebraic extensions of normed algebras, Proc. Amer. Math. Soc., 7 (1956), 203-210.

2. D. T. Brown, Galois theory for Banach algebras, Pacific J. Math., 30 (1969), 577-599.

3. L. N. Childs, On covering spaces and Galois extensions, Pacific J. Math., 37 (1971), 29-33.

4. I. Craw, Commutative abelian Galois extensions of a Banach algebra, J. Functional Analysis, 27 (1978), 170-178.

5. F. De Meyer and E. Ingraham, Separable Algebras Over Commutative Rings, Lecture Notes in Math., Vol. 181, Springer Verlag, 1971.

6. G. Leibowitz, Lectures on Complex Function algebras, Scott-Foresman, 1970.

7. J. A. Lindberg, Jr., Algebraic extensions of commutative Banach algebras, Pacific J. Math., 14 (1964), 559-583.

8. Homomorphisms of Banach algebras into Arens-Hoffman extensions of a semi-simple algebra, J. London Math. Soc., 13 (1976), 111-121.

9. A. Magid, Algebraically separable extensions of Banach algebras, Michigan Math. J., 21 (1974), 137-143.

10. C. E. Rickart, Natural Function Algebras, Universitext, Springer-Verlag, 1979. 
11. E. L. Stout, The theory of uniform algebras, Bogden and Quigley, Tarritown (1971). 12. J. Verdera, On finitely generated projective extensions of Banach algebras, to appear in Proc. Amer. Math. Soc.

13. B. M. Weinstock, Zero-sets of continuous holomorphic functions on the boundary of a strongly pseudoconvex domain, J. London Math. Soc., 18 (1978), 484-488.

14. O. Zarisky and P. Samuel, Commutative Algebra, Vol. I, Springer-Verlag, 1958.

Received October 1, 1980 and in revised form March 4, 1981.

UNIVERSITAT BARCELONA

GRAN VIA 585

Barcelona, Spain 



\section{PACIFIC JOURNAL OF MATHEMATICS}

\section{EDITORS}

DONALD BABBITT (Managing Editor)

University of California

Los Angeles, California 90024

Hugo RossI

University of Utah

Salt Lake City, UT 84112

C. C. MOORE and ARTHUR Agus

University of California

Berkeley, CA 94720

\section{J. DUGUNDJI}

Department of Mathematics University of Southern California Los Angeles, California 90007

R. FinN and J. MILGRAM Stanford University Stanford, California 94305

\section{ASSOCIATE EDITORS}
R. ARNES
E. F. BeCKENBACH
B. H. Neumann
F. WOLF
K. YosHIDA

\section{SUPPORTING INSTITUTIONS}

UNIVERSITY OF ARIZONA

UNIVERSITY OF BRITISH COLUMBIA

CALIFORNIA INSTITUTE OF TECHNOLOGY

UNIVERSITY OF CALIFORNIA

MONTANA STATE UNIVERSITY

UNIVERSITY OF NEVADA, RENO

NEW MEXICO STATE UNIVERSITY

OREGON STATE UNIVERSITY
UNIVERSITY OF OREGON UNIVERSITY OF SOUTHERN CALIFORNIA STANFORD UNIVERSITY UNIVERSITY OF HAWAII UNIVERSITY OF TOKYO UNIVERSITY OF UTAH WASHINGTON STATE UNIVERSITY UNIVERSITY OF WASHINGTON 


\section{Pacific Journal of Mathematics}

Vol. 100, No. $1 \quad$ September, 1982

Charalambos D. Aliprantis, Owen Sidney Burkinshaw and M. Duhoux,

Compactness properties of abstract kernel operators $\ldots \ldots \ldots \ldots \ldots \ldots 1$

Roger C. Alperin, Locally compact groups acting on trees .............23

Robert F. Brown, Real homology of Lie group homomorphisms ......... 33

Karen Chase, Maximal groups in sandwich semigroups of binary relations . . 43

W. Wistar (William) Comfort and T. Soundararajan, Pseudocompact

group topologies and totally dense subgroups $\ldots \ldots \ldots \ldots \ldots \ldots \ldots 61$

M. Ferri and C. Gagliardi, Crystallisation moves $\ldots \ldots \ldots \ldots \ldots \ldots \ldots$

Kenneth R. Goodearl, Directly finite aleph-nought-continuous regular

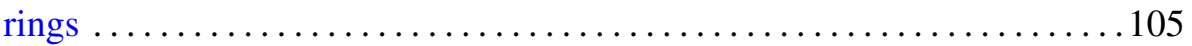

Edward Lewis Green, On the representation theory of rings in matrix

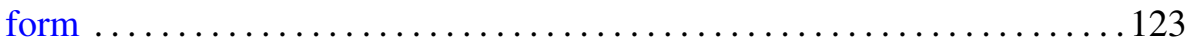

Walter Hengartner and Glenn E. Schober, Interpolation, continuation, and



Kenneth Kunen and Haskell Paul Rosenthal, Martingale proofs of some geometrical results in Banach space theory $\ldots \ldots \ldots \ldots \ldots \ldots \ldots \ldots \ldots \ldots$

Brian William McEnnis, Shifts on indefinite inner product spaces. II . . . . . 177

Roman Pol, Note on the spaces $P(S)$ of regular probability measures whose topology is determined by countable subsets $\ldots \ldots \ldots \ldots \ldots \ldots \ldots \ldots 185$

Joan Manuel Verdera Melenchón, Finitely generated projective extensions

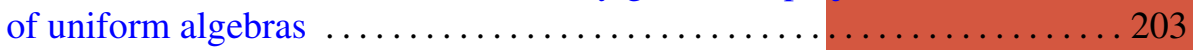

Cheng Ye You, Fixed point classes of a fiber map .................. 217 